\title{
LE MICROPLANCTON DE LA RIVIÈRE D'AURAY
}

\author{
par Gérard Paulmier
}

Le microplancton des régions ostréicoles de Bretagne fait régulièrement l'objet de récoltes effectuées en divers secteurs caractéristiques, tels que la Vilaine et la rivière d'Auray sur la côte

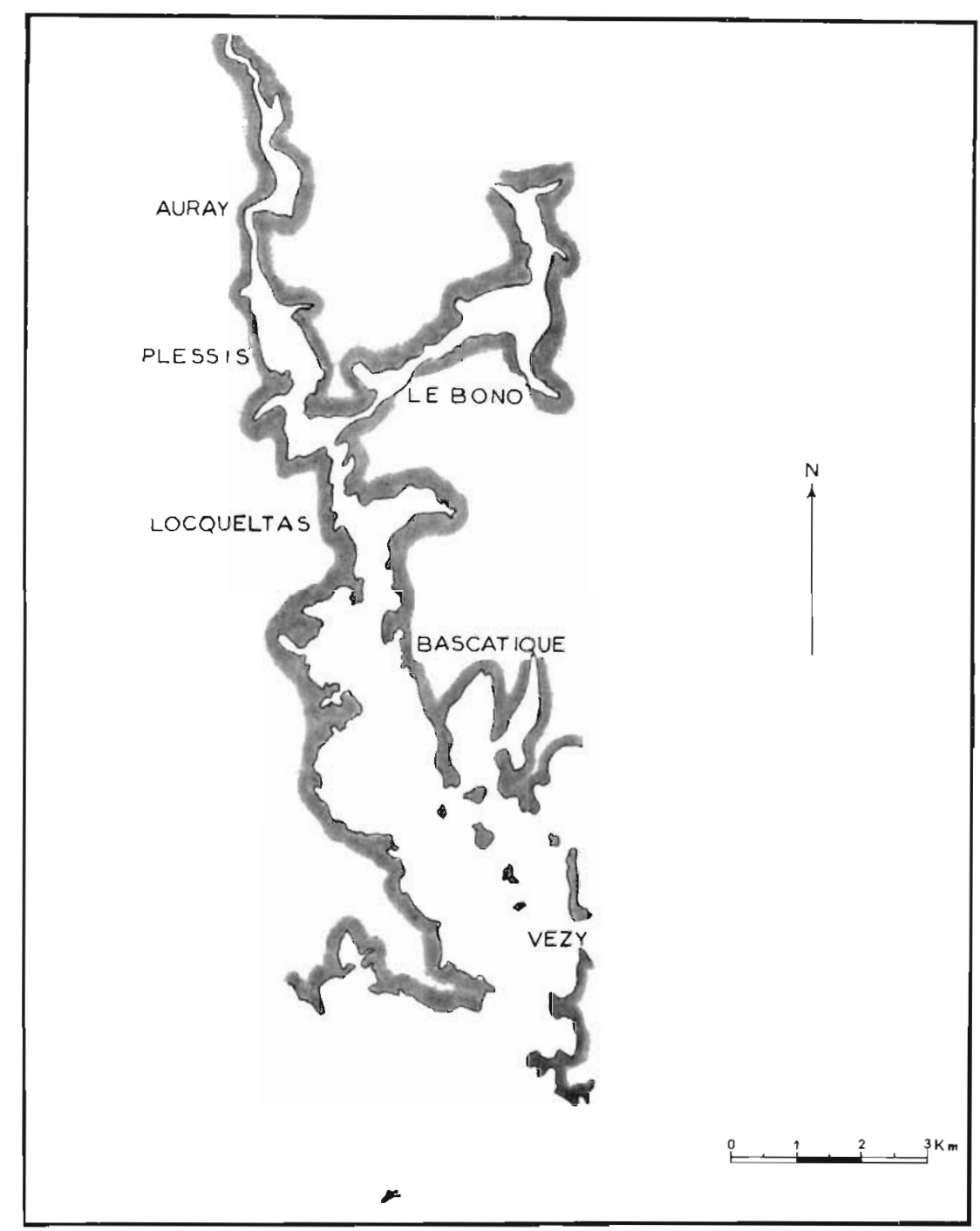

FIG. 1. - La rivière d'Auraly : stations de péches planctoniques

sud, la Penzé et la rivière de Morlaix sur la côte nord. Les examens portent sur la composition et les fluctuations des populations microplanctoniques et le dénombrement des principaux organismes. Simultanément on mesure certains facteurs physico-chimiques tels que température, salinité, $\mathrm{pH}$, 

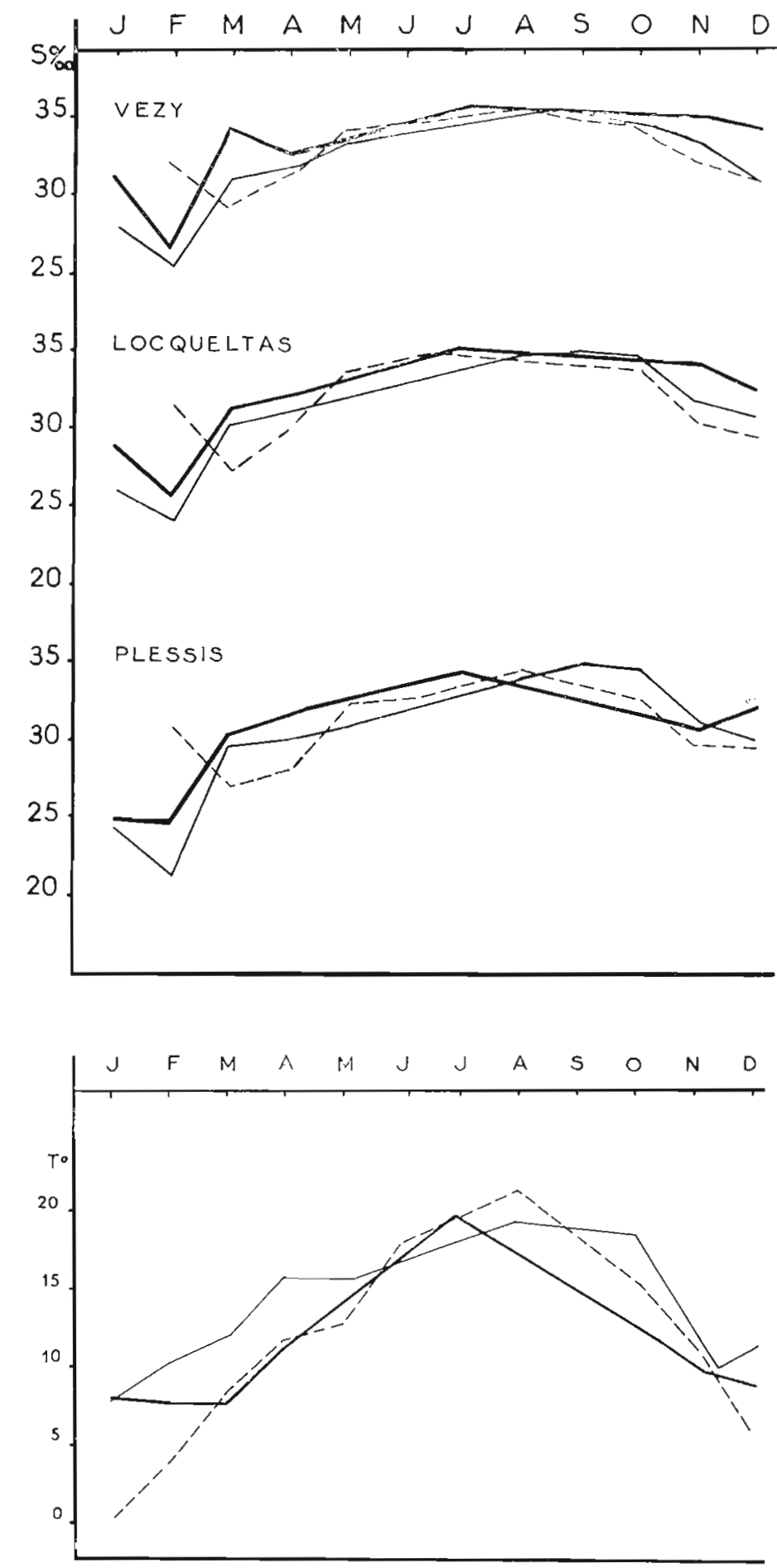

FIG. 2 et 3. - Températures de l'eau en ribière d'Auray (en haut), et salinités au fond daval (Vezy) en amont (Plessis) (en bas); trait plein. maigre 1961, gras 1962, tireté 1963. 
qui peuvent agir, comme les marées ou les courants, sur la distribution d'organismes dont la qualité et la quantité peuvent influencer la croissance, l'engraissement ou la reproduction des huitres ou moules, cultivées dans ces régions.

Nous examinerons particulièrement dans cette note le microplancton de la rivière d'Auray récolté en 1961, 1962 et 1963. Nos observations complèteront les travaux effectués antérieurement dans le même secteur par BORde (1938) (1) ct MarTeIL (1960) (1) qui les avaient limités soit à une période d'une année, soit à un groupe plus ou moins important d'organismes.

\section{Méthodes.}

Le plancton était recueilli une fois par mois au moyen d'un filet standard n' 200 , traîné en surface au moment de l'étale de pleine mer des marées de morte-eau, en diverses stations échelonnées de l'embouchure jusqu'à l'amont de la rivière d'Auray (fig. 1). L'examen était qualitatif et quantitatif, le dénombrement étant effectué sur des échantillons répartis sur plaque quadrillée selon le principe de la méthode de HeNSEN (1887). Les chiffres rapportés correspondent, conventionnellement, au nombre d'organismes théoriquement récoltés dans un trait de pêche d'une durée de quinze minutes. Ils n'ont qu'une valeur indicative.

\section{Le milieu.}

Marteil (1960) a déjà fait connaitre les conditions habituelles de courant, de température, de salinité, etc., en rivière d'Auray. Nous ne soulignerons ici que les particularités de certains de ces Facteurs pendant la période envisagée.

\section{Température.}

Le réchauffement printanier, précoce en 1961, survint plus tardivement en 1962 et 1963 (tabl. 1). Les températures maximales voisines de $21^{\circ}$, furent relevées chaque fois en juillet-aoùt (fig. 2).

\begin{tabular}{|c|c|c|c|c|}
\hline ANNÉES & Février & Mars & Avril & Mai \\
\hline$\ldots \ldots$ & $10^{\circ} 1$ & $11^{\circ} 7$ & $15^{\circ} 4$ & $15^{\circ} 3$ \\
\hline 1962 & $7^{\circ} 4$ & $7^{\circ} 4$ & $10^{\circ} 6$ & $14^{\circ}$ \\
\hline 1963 & $3^{\circ} 2$ & $8^{\circ}$ & $11^{\circ} 5$ & $12^{\circ} 5$ \\
\hline
\end{tabular}

TaBL. 1. - Températures mensuelles de l'eau (1961-1963).

Cependant, le phénomène le plus remarquable de cette période fut la rigueur prolongée de l'hiver 1962-1963 qui provoqua de profondes modifications au sein des masses d'eau, agissant sur le comportement des divers organismes benthiques et littoraux et perturbant la production ostréicole morbihannaise (Marteil, 1963. Science et Pêche n 115).

Salinités.

Les variations de salinité de l'amont à l'aval et de la surface au fond peuvent être, on le sait, très importantes. On l'a constaté à nouveau en février 1961, où la salinité était de 6,5\%o en surface et de $21,3 \%$ à la profondeur de 6 mètres à la station amont, alors quelle était de $23,9 \%$ en surface et de $25,3 \%$ à la profondeur de 20 mètres, en aval.

De 1961 à 1963, les valeurs de ce facteur ne se sont pas écartées des valeurs habituelles; la dessalure se fit surtout sentir en période de crue (en février 1961 et 1962, en mars 1963) en amont et en surface, tandis que la salinité atteignait des valeurs égales ou supérieures à $35 \%$, durant la période estivale (fig. 3).

$p H$.

Les mesures effectuées occasionnellement ont donné un $\mathrm{pH}$ compris entre 8,0 et 8.4 pouvant descendre accidentellement à 7,7 dans la partie amont la plus exposée à la dessalure.

(1)Reu. Trav. Off. Pêches marit., t. 11 f. 4 et t. 24 f. 3. 


\section{Le plancton.}

\section{Phytoplancton.}

Le phytoplancton est en partie composé de diverses microalgues telles que Halosphaera viridis Schmirz (commune de l'automne au printemps) et de protococcales d'eau saumâtre ou douce : Scenedesmus quadricauda (TuRp) BreBISSON et Pediastrum sp.

Cependant les organismes les plus nombreux appartiennent aux deux grands groupes des Diatomées et des Dinoflagellés.

\section{Diatomées.}

181 espèces, 2 variétés, 3 formes, appartenant à 66 genres ont été identifiées (tabl. 4, à la fin). Cette liste n'est d'ailleurs pas limitative.

\section{Distribution saisonnière.}

Au printemps, première période de grande richesse phytoplanctonique, quatre diatomées ont une importance majeure en raison du nombre d'individus par espèce : Skeletonema costatum Cleve pré-printanière, Nitzschia seriata Cl., Rhizosolenia shrubsolei BRIGHTwELl, Chaetoceros curvisetus $\mathrm{CL}_{\mathrm{L}}$ Il en est beaucoup d'autres et parmi les plus remarquables nous citerons : Guinardia flaccida H. Peragallo, Cerataulina bergonii H. Per., Leptocylindricus danicus Cl., Chaetoceros laciniosus SchuTt, Rhizosolenia setigera Brightw., Dytilum brightwellii Gran, etc.

En été, si l'abondance diminue, la population diatomique reste très variée. On peut distinguer plusieurs espèces prédominantes : Rhizosolenia alata BrighTw., R. styliformis BrigHTw., Chaetoceros eibenii Cl., Bacteriastrum varians Lauder, Coscinodiscus centralis Ehrengerg, C. concinnus W. SMITH.

L'automne est également propice au développement végétal. Les espèces sont variées et les individus nombreux. La dominance de certains organismes y est moins accusée qu'au printemps. Une des causes de l'abondance des diatomées en cette saison est constituée en partie du moins, par un second maximum de certaines espèces printanières, telles que Rhizosolenia shrubsolei et Nitzschia seriata. Parmi les autres principaux éléments observés en automne, nous trouvons Chaetoceros decipiens Cl., C. affinis CL., C. danicus CL., Rhizosolenia styliformis Brightw., Stephanopyxis palmeriana Greville, Coscinodiscus excentricus Ehr., Biddulphia sinensis Grev.

En hiver, l'abondance comme la diversité du phytoplancton sont variables; les espèces sont plus petites ou filamenteuses. Le nombre des diatomées benthiques croît par rapport aux saisons précédentes. Les genres Thalassiosira $\mathrm{Cl}$. et Biddulphia GRAY sont très caractéristiques avec comme espèce principale : Thalassiosira decipiens JORGENSEN et Biddulphia aurita HuSTEDT. Les mélosirées sont communes.

En résumé chaque période est caractérisée par la présence de quelques espèces plus ou moins saisonnières, apparaissant en plus ou moins grand nombre suivant l'année considérée. Il convient d'ajouter que beaucoup d'autres diatomées se rencontrent en permanence durant toute l'année : ce sont pour la plupart des éléments semi-benthiques ou benthiques qui abondent plus particulièrement en amont de la rivière. On peut les considérer de par leur constance, comme faisant partie intégrante du plancton. Les genres Pleurosigma W. SM. et Nitzschia Hassal, groupant plusieurs espèces, sont nettement prédominants.

\section{Dinoflagellés.}

48 espèces, 3 variétés, représentant 13 genres ont été reconnues jusqu'à ce jour (liste ci-après).

\section{Distribution saisonnière.}

Comme pour les diatomées, la distribution et l'abondance des dinoflagellés varient selon le lieu et la saison ; les peuplements les plus diversifiés et les plus abondants se situent généralement au printemps et en automne. 
Au printemps, la population de dinoflagellés est composée en majeure partie de Ceratium fusus, C. Furca, C. tripos, C. intermedium. C. macroceros, de Peridinium oblongum, P. diabolus, $P$. conicum, $P$. steini, et de Prorocentrum micans. On rencontre aussi Phalacroma rotundatum et Dinophysis acuminata.

En été on trouve de nombreuses formes, Peridinium claudicans, Ceratium longipes et Pyrophacus horologium étant les plus communs.

En automne les dinoflagellés prospèrent. On retrouve des espèces printanières et. en plus, Peridinium depressum, $P$. pentagonum. Ceratium candelabrum. Dinophysis tripos et caudata, ainsi que des Goniaulax (digitale, polyedra).

Enfin, l'hiver est caractérisé par la présence de petites formes, notamment : Glenodinium mucronatum, Exuviella marina, Peridinium ovatum.

Nous mentionnerons également les silicoflagellés représentés par deux espèces, communes au printemps et en automne : Distephanus speculumEHr. et Dictyocha fibula EHr. et les ébriediens dont une seule espèce hante nos parages : Ebria tripartita Schumann.

La plupart des organismes rencontrés sont électifs ou préférants d'une saison; deux espèces seulement se rencontrent communément toute l'année: Ceratium fusus et C. furca.

\author{
PROROCENTRIDAE \\ Exuviella marina CIENK \\ Prorocentrum sp. EHR. \\ Prorocentrum micans EHR. \\ DINOPHYSIDAE \\ Phalacroma rotundatum $\mathrm{CL}_{\mathrm{L}}$. \\ Phalacroma acutum SchuTT \\ Dinophysis acuminata KofolD \\ Dinophysis acuta EHR. \\ Dinophysis tripos Gourret \\ Dinophysis caudata KENT \\ GYMNODINIDAE \\ Amphidinium sp. $\mathrm{Cl}$. \\ Gyrodinium pavillardi Brecheler \\ NOCTILUCIDAE \\ Noctiluca milliaria Suriray \\ GLENODINIOPSIDAE \\ Pyrophacus horologium Schiller \\ GLENODINIDAE \\ Glenodinium mucronatum W. CONRAD \\ Peridiniopsis asymetrica MangIN \\ Diplopsalopsis orbicularis PauLSEN \\ PERIDINIDAE \\ Peridinium mite Pavillard \\ Peridinitum steini JORG. \\ Peridinium piriforme Paulsen \\ Peridinium diabolus CL. \\ Peridinium ovatum Pouchet \\ Peridinium pellucidum Bergh \\ Peridinium divergens EHR. \\ Peridinium crassipes Kofold \\ Peridinium conicum Gran
}

Peridinium leonis Pavillard

Peridinium pentagonum GraN

Peridinium conicoides

Peridinium willei

Peridinium punctulatum

Peridinium depressum BAILEY

Peridinium oceanicum VANHÖFfEN

Peridinium oblongum $\mathrm{C}_{\mathrm{L}}$.

Peridinium claudicans PaUlsen

Peridinium simplex Gran et BraArud

GONIALILACIDAE

Goniaulax digitale PoucheT

Goniaulax polyedra STEIN

Goniaulax spinifera Diesing

CERATIDAE

Ceratium candelabrum STEIN

Ceratium furca EHR.

Ceratium lineatus $\mathrm{E}_{\mathrm{HR}}$.

Ceratium falcatum KoFoid

Ceratium fusus EHR.

Ceratium macroceros EHR.

Ceratium tripos (Muller) Nitzsche

Ceratium tripos f. atlantica Ostenfend

Ceratium tripos f. subsalsa Ost.

Ceratium tripos f. hiemale Paulsen

Ceratium bucephalum $\mathrm{CL}_{\mathrm{L}}$.

Ceratium intermedium JORG.

Ceratium batavum PAULSEN

Ceratium longipes BAILEY

DINOCOCCIDAE

Pyrocystis lunula SCHutr

Pyrocystis elegans Pavillard 


\section{Le zooplancton.}

En dehors des stades larvaires de mollusques, d'huitres notamment, de crustacés, de poissons, et de divers hôtes de la rivière, régulièrement trouvés mais que nous n'étudions pas ici, les organismes animaux les plus remarquables appartiennent à plusieurs groupes holoplanctoniques : Tintinnides, Rotifères, Cladocères, Copépodes. Mais en raison du procédé de pêche et des possibilités natatoires de beaucoup de ces animaux (copépodes surtout), la quantité récoltée ne représente pas exactement la totalité de la population zooplanctonique. Les dénombrements se rapportant particulièrement au macroplancton sont donc donnés sous réserve.

\section{Tintinnides.}

Ils sont représentés par de nombreuses espèces dont toutes n'ont pu être identifiées.

\section{Oligot riches.}

\section{CODONELLIDAE}

Tintinnopsis beroidea STEIN

Tintinnopsis campanula EHR.

Tintinnopsis bütschlii DADAY

Tintinnopsis cylindrica DADAY

Tintinnopsis lobiancoi DADAY

Codonella sp. FoL

\section{CODONELLOPSIDAE}

Stenosemella nivalis MEUNIER

\author{
METACYLINAE \\ Helicostomella subulata EHR. \\ CYTTAROCY LIDAE \\ Cyttarocylis sp. FoL \\ PTYCHOCYLIDAE \\ Favella serrata MöBius \\ Favella markuzowskii DADAY \\ TINTINNIDAE \\ Tintinnus sp. Schrank
}

Les tintinnides jouent un grand rôle dans le métabolisme de la rivière, du fait de leurs pullullements périodiques (550000 en février et 315000 en octobre 1963), qui suivent fréquemment de fortes poussées phytoplanctoniques et zooplanctoniques. Ces organismes omnivores se nourrissent de proies minuscules (Tregouboff, 1957) (1), limitant ainsi le développement du microplancton, avant d'être ingérés eux-mêmes ou de tomber sur le fond pour s'y décomposer, quand la nourriture se fait plus rare. Parmi les formes remarquables par leur pullullation, Tintinnopsis campanula, Stenosemella nivalis, Codonella sp. occupent une place de choix.

\section{Rotifères.}

Deux espèces principales, appartenant au genre Synchaeta EHR., sont nettement saisonnières de mars à juin, proliférant souvent en avril-mai et parfois en juin (115000 en avril 1962 et 154000 en avril 1963). D'autres rotifères comme Keratella quadrata quadrata Mueller sont indicateurs d'apport d'eau douce et peuvent se rencontrer en amont de la rivière (station du Plessis. sal. $21,1 \%$ le 3 mai 1963).

\section{Cladocères.}

Cinq espèces les représentent.

$$
\begin{aligned}
& \text { Penilia avirostris Dana } \\
& \text { Podon leuckarti S.O. Sars } \\
& \text { Podon intermedius Lill Jeborg }
\end{aligned}
$$

Evadne nordmanni Loven Evadne spinifera MulLeR

Penilia avirostris apparaît régulièrement en septembre, son passage dans la rivière est fugace. C'est l'époque où les fortes salinités $(34,9-35,2 \%)$ coïncident ici avec des températures encore élevées $\left(18^{\circ}-18^{\circ} 8\right)$. Cependant cette espèce semble s'accommoder de conditions diverses et serait euryhaline (Rose, 1957). Les quatre autres espèces citées apparaissent en avril-mai, et leur période optimale se situe en été. Podon lauckarti semble être plus largement distribué dans le temps.

(1) TrÉgoubopr (G.) et Rose (M.). Manuel de planctonologie méditerranéenne, Paris. CNRS. 


\section{Copépodes.}

Les copépodes rencontrés sont relativement nombreux.

CALANIDAE

Calanus finmarchicus GunNERUS

Paracalanus parvus Claus

Pseudocalanus elongatus BOECK

\section{CENTROPAGIDAE}

Centropages typicus KRÖYER

Centropages hamatus LiLI.JeB.

Isias clavipes BOECK

Metridia longa LubBock

Temora longicornis BAIRD

Temora stylifera DANA

Eurytemora hirundoides NoRDQuIST

Pontella sp.

Pontella lobiancoi Canu

Parapontella brevicornis LuвB.

Anomalocera patersoni TeMPLETON

Labidocera wollastoni LuBB.

Acartia clausi Giesbrecht

Acartia longiremis LiLljeB.

Acartia discaudata Giesbr.

Acartia grani SARS

\author{
ONCAEIDAE \\ Oncæa sp. \\ Oncrea media Giesbr. \\ Onceea conifera GIESBR. \\ OITHONIDAE \\ Oithona plumifera BAIRD \\ Oithona similis CuS. \\ HARPACTICOIDAE \\ Euterpina acutifrons DANA \\ Clytemrestra rostrata BRADY \\ Halithalestris croni KRÖYER \\ Amynome sphaerica Crs. \\ Microsetella norvegica BOECK \\ PELTIDIIDAE \\ Scutellidium tisboides CLS. \\ CORYCAEIDAE \\ Corycaeus anglicus LuBB. \\ Corycaeus speciosus DANA \\ MONSTRILLIDAE \\ Monstrilla sp. \\ Cymbasoma longispinosum GIESBR.
}

Les copépodes représentent la fraction la plus importante, en nombre (en mai 1961, on dénombre 250000 individus en amont, appartenant au genre Acartia) et surtout en volume, du zooplancton, en presque toutes circonstances. L'espèce ou les espèces dominantes ne sont pas les mêmes suivant la saison et le lieu. Beaucoup d'entre elles se rencontrent toute l'année, entre autres : Acartia clausi, A. longiremis, Temora longicornis, Euterpina acutifrons, Corycaeus anglicus.

D'autres sont trouvées plus irrégulièrement: Centropages hamatus, Oithona plumifera.

Enfin, quelques-unes sont nettement saisonnières,

printanières: Acartia discaudata, Isias clavipes, Centropages typicus,

estivales : Acartia grani, Parapontella brevicornis, Labidocera wollastoni,

automnales : Oncaea media, Temora stylifcra.

Outre les diverses espèces pélagiques citées, on trouve des copépodes benthiques dont le nombre va croissant d'aval en amont.

\section{La distribution du plancton et les facteurs physico-chimiques.}

Les observations précédentes ont déjà mis en évidence le caractère permanent ou saisonnier de certaines espèces ainsi que l'importance inégale des poussées planctoniques. C'est ainsi que la floraison vernale est bien supérieure à celle des autres saisons (fig. 4), que dès le début du printemps, on assiste à des éclosions très nombreuses d'animaux et qu'en êté, les stades larvaires apparaissent en plus ou moins grandes quantités (fig. 5). Cependant une étude plus poussée permet d'établir des relations plus précises entre le comportement et la distribution de la microflore ou de la microfaune et certains facteurs physico-chimiques de ce milieu d'estuaire. 


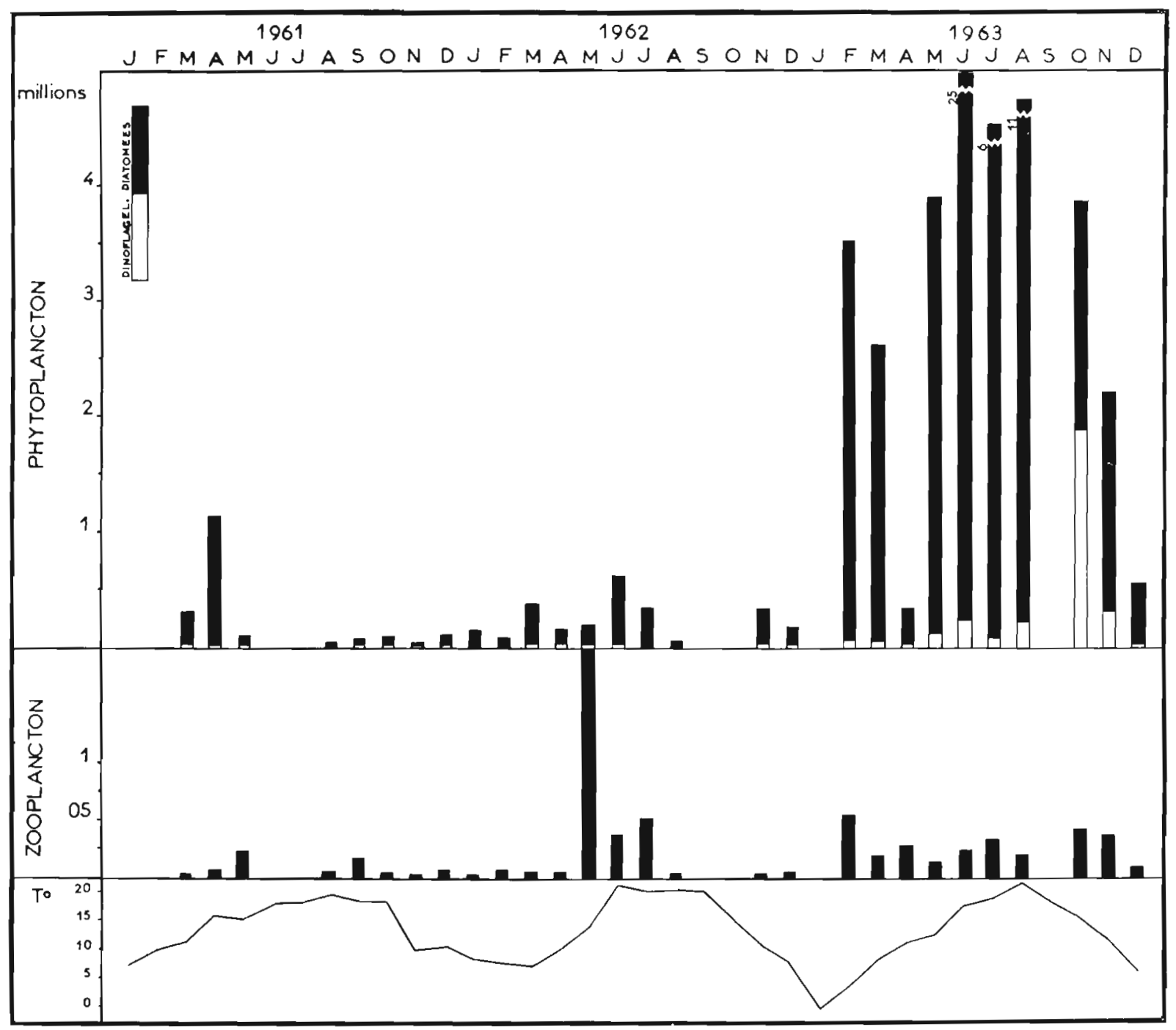

Fig. 4. - Cycle du phytoplancton (diatomécs en noir, et dinoflagellés en blanc) et du zooplancton, en fonction de la température, de 1961 à 1963.

Les marées et les courants.

On constate une accumulation des organismes en certains points de la rivière, notamment à hauteur de la station située dans le tiers supérieur de l'estuaire (Locqueltas); ainsi le 24 juillet 1962 la répartition était la suivante:

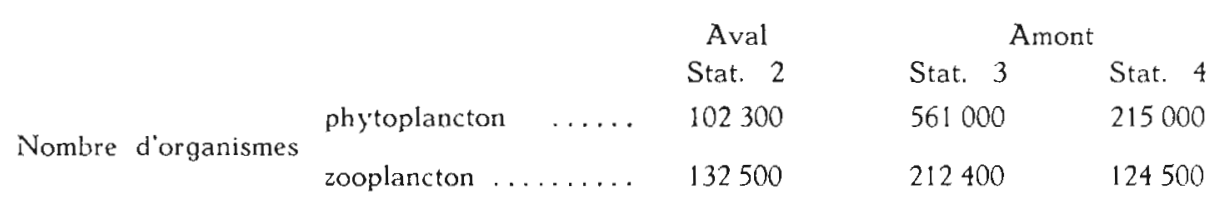

Marteil (1960) avait déjà constaté une augmentation du nombre des larves pélagiques d'Ostrea edulis dans les parties médianes et supérieures de la rivière d'Auray, due, selon lui, à l'action des courants. Le phénomène on le voit est valable pour les différents organismes planctoniques.

Les marées de vive-eau, par le brassage des fonds, entrainent la remise en suspension d'éléments 
benthiques, organiques et minéraux. Cependant, si le volume brut de plancton recueilli dans ces conditions est plus élevé du fait surtout de la présence d’éléments minéraux, le nombre des organismes planctoniques pélagiques est souvent moins grand qu'en période de morte-eau.

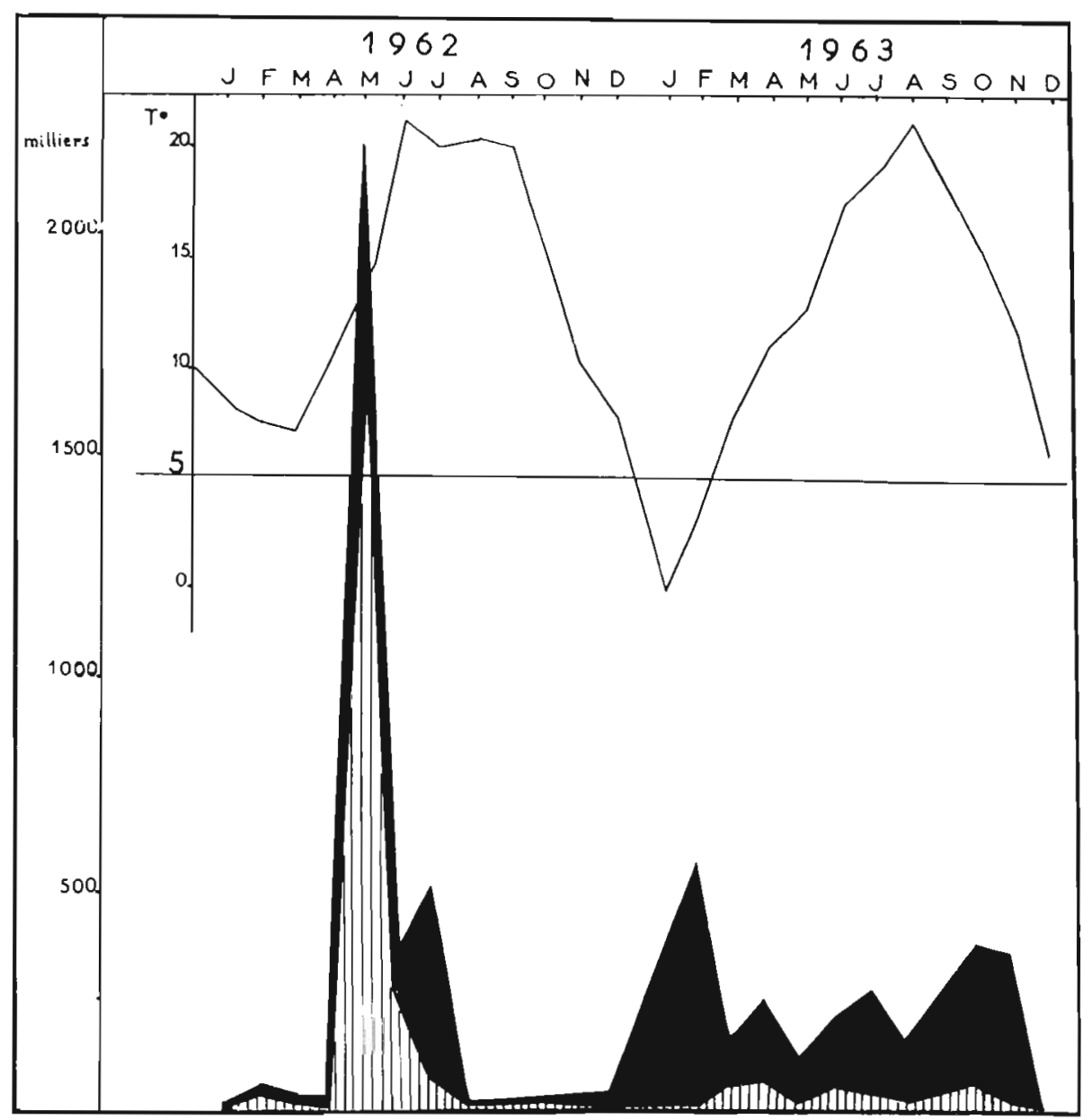

FiG. 5. - Cycle du zooplancton : stades larvaires (hachures) et holoplancton (en noir), en 1962 et 1963.

Le tableau 2 indique les volumes de plancton recueillis à différents coefficients de marée dans une même station.

\begin{tabular}{|c|c|c|c|c|c|}
\hline \multicolumn{3}{|c|}{ Vive-eau } & \multicolumn{3}{|c|}{ Morte-eau } \\
\hline Date & Coeff. & Volume (ml) & Date & Coeff. & Volume (ml) \\
\hline $\begin{array}{l}22-\mathrm{V} \\
1-\mathrm{VI} \\
5-\mathrm{VI}\end{array}$ & $\begin{array}{l}85 \\
91 \\
85\end{array}$ & $\begin{array}{l}11, \\
11,25 \\
9,5\end{array}$ & $\begin{array}{c}25-\mathrm{V} \\
8-\mathrm{VI} \\
15-\mathrm{VI}\end{array}$ & $\begin{array}{l}64 \\
57 \\
63\end{array}$ & $\begin{array}{l}8.25 \\
5.5 \\
2.5\end{array}$ \\
\hline
\end{tabular}

TABL. 2. - Volumes de plancton recueillis selon différents coefficients de marée sur une même station, en 1962.

Ne peut-on penser que les éléments pélagiques sont alors dispersés dans un volume d'eau plus important ou qu'ils sont entraînés vers le fond par les particules minérales en suspension? 


\section{La salinité et la température.}

Certaines espèces sont présentes, en permanence, dans les diverses stations de l'estuaire, les unes sont pélagiques, telles Chaetoceros decipiens, C. danicus, Bacillaria paradoxa, Striatella unipunctata (ces deux dernières plutôt semi-pélagiques). Thalassiothrix nitzschioides, Acartia clausi. A. longiremis, Euterpina acutifrons, Temora longicornis et certains Tintinnides ;

les autres sont benthiques ou épiphytes telles Pleurosigma sp., Navicula sp., Nitzschia sp., Surirella sp., Cocconeis, Halithalestris croni et divers autres Harpacticidés. Du fait de leur permanence, tous ces organismes peuvent être considérés comme eurythermes et euryhalins.

Nombre d'éléments n'apparaissent que dans la partie supérieure de la rivière, dans les eaux fortement dessalées à la suite des crues. Ce sont des formes d'eau saumâtre, voire d'eau douce: Scenedesmus quadricauda, Pediastrum sp., Dinobryon sp., Synedra radians et S. fulgens, Keratella quadrata quadrata, Eurytemora hirundoides. Ces organismes ne sont retrouvés quaccidentellement dans la partie aval de l'estuaire où les salinités sont plus élevées.

Il est plus difficile de déterminer le facteur prépondérant dans l'apparition des espèces franchement saisonnières, températures et salinités s'élevant ou s'abaissant souvent de façon concomitante. Certaines espèces estivales ou automnales ne prospèrent cependant que pour des températures supérieures à $16^{\circ} 5$, leur production maximale coïncidant généralement avec une température égale ou supérieure à 20․ C'est le cas de Chaetoceros teres, C. socialis, C. lorenzianus, C. brevis, Bacteriastrum varians, Goniaulax polyedra et G. spinifer. Phalacroma rotundatum. Dinophysis acuminata, Parapontella brevicornis et Acartia grani. Tous ces organismes sont alors largement distribués en rivière.

Dans le même temps d'autres formes sont particulièrement concentrées en aval; elles n'apparaissent dans la partie supérieure de la rivière que fortuitement et pour peu de temps. Leur aire de distribution semble ici limitée par l'isohaline de $34,5 \%$. Ces formes comprennent Rhizosolenia styliformis, Coscinodiscus sp., Peridinium depressum et P. oceanicum, Oithona plumifera, Temora stylifera. Centropages typicus. Labidocera wollastoni, Pseudocalanus elongatus. C'est à ce moment qu'on trouve aussi en grand nombre Autolytus sp., des Chaetognathes et des Appendiculaires. Par les conditions de leur apparition et de leur aire de distribution, ces organismes pourraient être considérés ici comme sténohalins, d’origine néritique océanique ou franchement océanique.

Parmi les espèces hivernales ou pré-printanières, certaines ne prospèrent parfaitement qu'aux basses températures, leur floraison dépendant semble-t-i] de la rigueur et de la durée du refroidissement. C'est le cas de Thalassiosira sp., Coscinosira polychorda et Biddulphia aurita, formes des mers arctiques et boréales (Marukawa, 1921 (1), Brunel, 1962). C'est ainsi que ces éléments se développèrent peu en 1961 et 1962 où les températures de l'eau ne descendirent pas au-dessous de $+7^{\circ}$ et se relevèrent rapidement. En revanche, pendant l'hiver 1962-1963 où la température descendit à $+3^{\circ}$ dans les chenaux et même au-dessous, dès le mois de janvier, pour ne dépasser $+5^{\circ} q^{\prime} u^{\prime}$ mars, les espèces considérées connurent un développement considérable (tabl. 3).

\begin{tabular}{|c|c|c|c|}
\hline Diatomées & Vezy & Bascatique & Plessis \\
\hline Biddulphia aurita & 3200000 & 5520000 & 11500000 \\
\hline Thalassiosira dectpiens .. & 22300 & 26500 & 95000 \\
\hline Coscinosira polychorda & traces & 139600 & 115000 \\
\hline
\end{tabular}

TABL. 3. - Répartition quantitative de quelques diatomées d'eau froide de l'aval (Vezy) vers lamont (Plessis), le 18.11.1963.

La répartition de Biddulphia aurita, en pourcentage total, pendant les trois années d'observation, met en valeur ce développement inhabituel,

$\begin{array}{rrrrr}\text { Année 1961: } & \text { hiver } 0,083 & \text { printemps traces, } & \text { été traces, automne } 0,004 \% \\ 1962: & 0,099 & 0,093 & 0,052 & 0.219 \\ 1963: & 97,128 & 0,227 & 0,413 & 1,682\end{array}$

(1) Bull. Inst. océanogr., Monaco, $\mathrm{n}^{\circ} 384$. 
Pendant le rude hiver 1962-1963, de nouvelles espèces apparaissent qui n'avaient pas été décelées jusqualors: Thalassiosita gravida, T. hyalina, T. nordenskioldii, Asterionella japonica, A. kariana, etc. La plupart de ces diatomées disparaissaient des eaux de la rivière d'Auray lorsque la température atteignait $15^{\circ}$, ou ne subsistaient qu'à l'état endémique.

\section{Les variations annuelles.}

Les variations qualitatives et quantitatives du microplancton étaient peu accusées en 1961 et 1962. Sa composition semblait même très voisine de celle des années 1935-1936 et 1956-1957 (pour les mois d'été). étudiées respectivement, bien que de façon moins détaillée par BORDE (1938) et Marteil (1960). Le caractère exceptionnel de l'année 1963 n'en sera que mieux souligné.

Nous avons déjà signalé le développement considérable des diatomées électives d'eaux froides pendant les premiers mois de 1963. Cette augmentation de la production phytoplanctonique ne fut cependant pas limitée à la période hivernale, les plus grands nombres de diatomées étant relevés en juin, juillet et août (fig. 4). Qualitativement, pendant toute l'année, de nouvelles espèces apparurent, qui n'avaient pas été inventoriées jusque-là; d'autres, rares ou mal représentées les années précédentes, proliférèrent au point de constituer le groupe le plus important (Chaetoceros).

Les populations de dinoflagellés furent également plus nombreuses et plus variées que de coutume, celles-ci suivant à peu près le même rythme biologique que les populations diatomiques, et en conséquence, le zooplancton fut lui aussi plus abondamment et plus régulièrement représenté chaque mois (fig. 5).

Les causes de cette pullulation ont été probablement très diverses. A l'origine, on trouve vraisemblablement le long et rigoureux refroidissement de janvier-février 1963 qui a pu, de façons très variées, provoquer un enrichissement des eaux en sels nutritjfs permettant d'abord le développement exceptionnel du phytoplancton et indirectement celui du zooplancton : renouvellement des eaux par des courants de convection créés par le refroidissement de la surface (Nathansohn, 1909) (1) sous l'action de vents persistants des secteurs nord et est, accompagné d'un éclairement inhabituel en cette saison, par des eaux plus riches du large; apports de matières organiques et minérales à la suite des mortalités importantes des animaux marins touchés par le froid, etc.

\section{Résumé el conclusion.}

L'inventaire du microplancton récolté en rivière d'Auray de 1961 à 1963 a permis d'établir le caractère permanent ou saiscnnier de nombreux organismes dont beaucoup sont inégalement distribués de l'aval vers l'amont.

Les marées et les courants, la température et la salinité sont des facteurs responsables de la rêpartition des micro-organismes. C'est ainsi que les formes d'eau douce ou d'eau saumâtre ne sont hatituellement rencontrées que dans la partie supérieure de la rivière soumise aux crues tandis que les formes océaniques sont plus abondantes dans les stations proches de l'embouchure.

Un abaissement prolongé de la température favorise en outre le développement de formes électives d'eaux froides. La composition du microplancton, son abondance relative et sa distribution en rivière d'Auray sont ainsi sous la dépendance de facteurs physico-chimiques, eux-mêmes conditionnés par le climat ou la topographie.

C'est à une modification brutale du climat, manifestée par le rigoureux hiver de 1963, que semblent pouvoir être attribués les changements profonds et durables constatés depuis lors dans la composition et l'abondance du microplancton de la rivière d'Auray.

Corrélativement, cette exceptionnelle richesse planctonique a favorisé, en 1963. la croissance des huitres plates (Ostrea edulis L.) sur les gisements naturels comme sur les parcs. Elle a, sans doute, infuencé aussi l'apparition ou la multiplication d'organismes considérés, à juste titre, comme compétiteurs ou prédateurs des mollusques (balanes, carcines, astéries) qui ont envahi à cette époque les zones ostréicoles du Morbihan.

(1) Bull. Inst. océanogr., Monaco, n" 140. 
Taßi.. 4. - Liste mensuelle des diatomées et algues en rivière d'Auray. en 1962 st 1963.

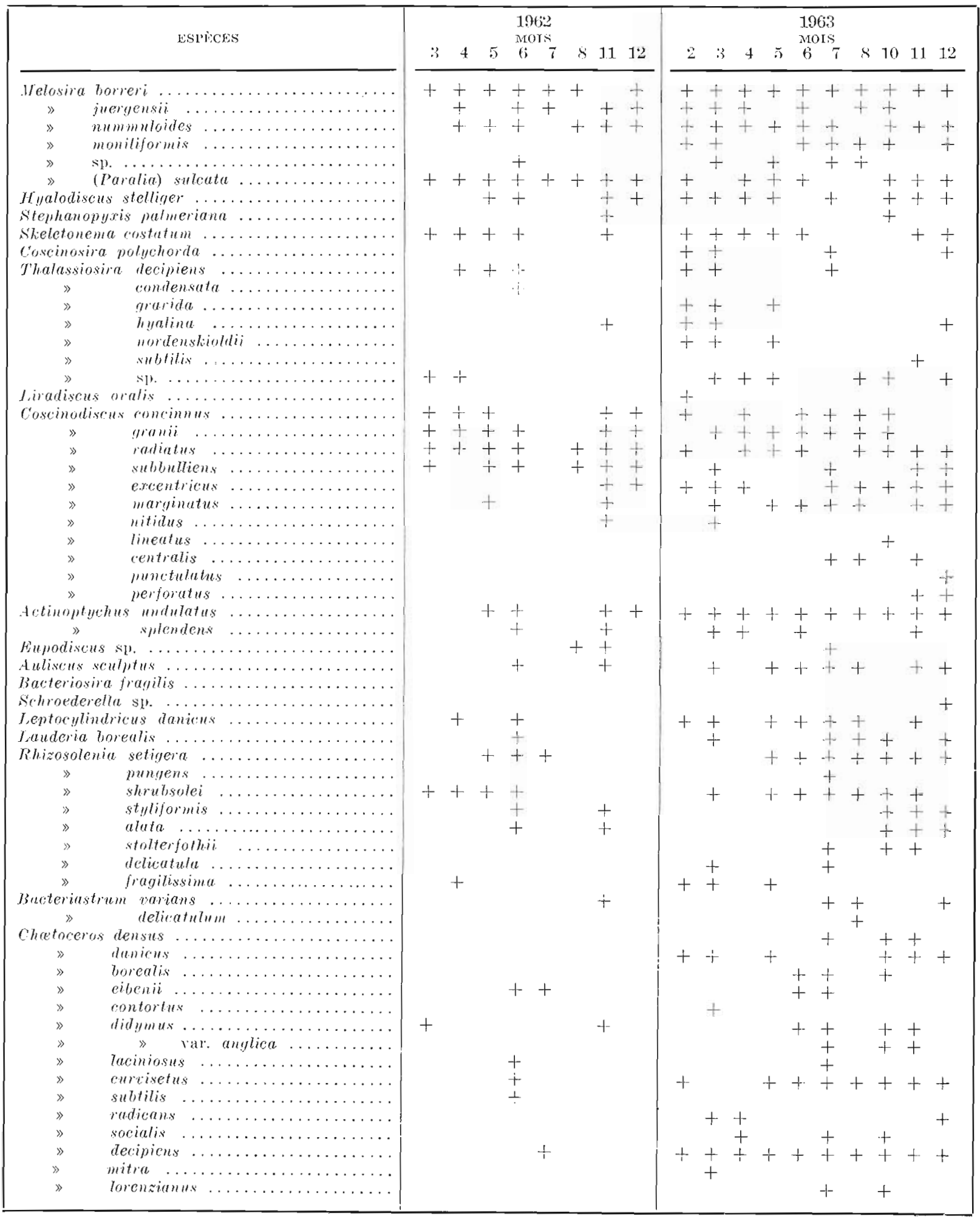


Tableau 4 (suite).

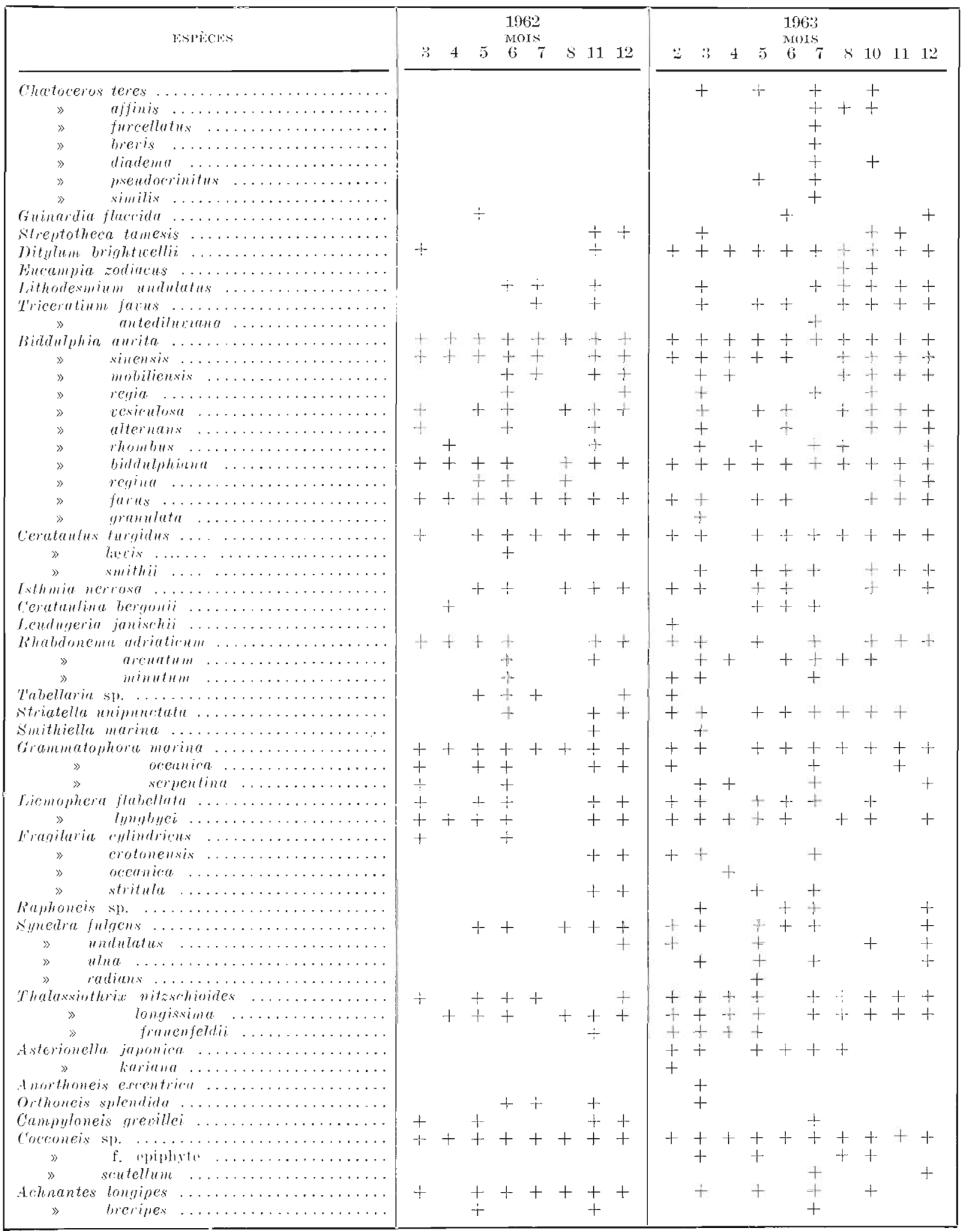


TABLEALI 4 (suite)

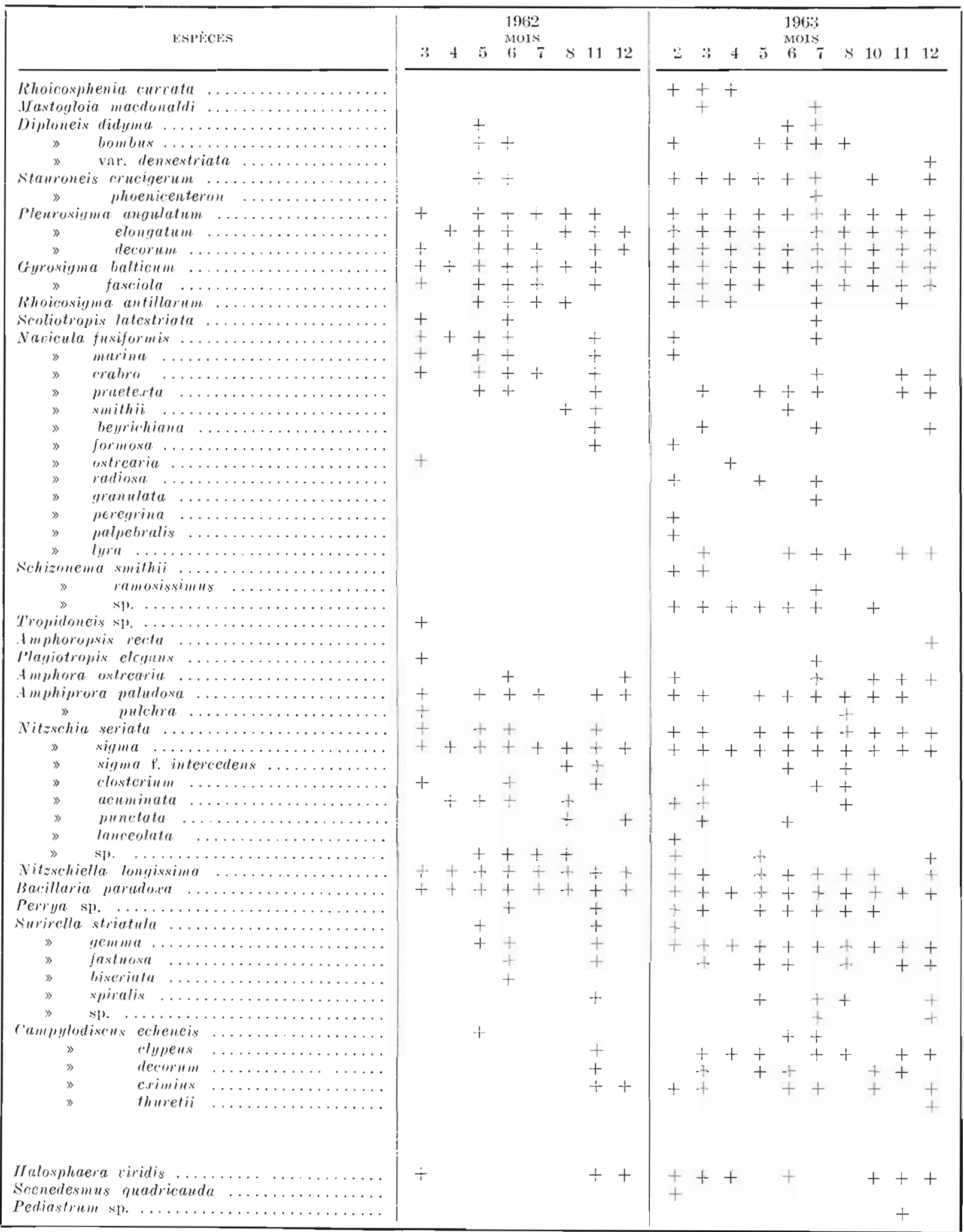

